\title{
Using a Lightweight Ontology of Heart Electrophysiology in an Interactive Web Application
}

\author{
Bernardo Gonçalves, Veruska Zamborlini, Giancarlo Guizzardi, José G. Pereira Filho \\ Computer Science Department \\ Federal University of Espírito Santo \\ (UFES), Vitória, Brazil
}

\{bgoncalves, veruska, gguizzardi, zegonc\}@inf.ufes.br

\begin{abstract}
Biomedical knowledge and data have been increasingly more explored by computer programs in providing support for human visualization of biomedical phenomena. Use scenarios of such graphical simulations range from physician's decision support to aid-learning in medical sciences. The knowledge and data representation often plays a prominent role in such biomedical applications. In this sense, this paper introduces the use of a lightweight ontology of heart electrophysiology in an interactive web application. We reflect on this ontology-based application to highlight some benefits achieved with a representation based on a combination of the semantic web languages OWL DL and SWRL. The application can be used to offer support for interactive learning in heart electrophysiology.
\end{abstract}

\section{Categories and Subject Descriptors}

H.3.5 [Information Storage and Retrieval]: Online Information Services - Web-based services; I.2.1 [Artificial Intelligence]:

Applications and Expert Systems - Medicine and Science; H.5.1

[Information Systems]: Information Interfaces and Presentation - Multimedia Information Systems.

\section{General Terms}

Design, Languages, Standardization, Experimentation.

\section{Keywords}

Ontology, Reasoning, OWL DL and SWRL, Web multimedia animations, Heart electrophysiology, ECG, Medical education.

\section{INTRODUCTION}

Biomedical applications are ever more common on account of recent developed technologies. They are used with several different purposes such as physician's decision support, knowledge management and aid-learning in medical sciences, among others. It can be argued, however, that the representation of knowledge and data in biomedical applications is often the bottleneck in the whole development phase of such applications. Indeed, suitably representing the biomedical knowledge and data is indeed a challenge, given their size and complexity.

In face of this, there has been a growing trend towards the use ontology as a representation formalism for the biomedical domain. This has been evidenced by the literature, e.g. [2], [7], [14]. Some of the motivations include: (i) ontological foundations have been successfully employed to help understanding specific domains of reality; (ii) there exists many formalisms for ontology representation which offer a sound support for automatic reasoning (e.g., different flavors of Description Logics (DLs) [1]); (iii) ontologies can be used as reusable artifact to affort reuse of knowledge across multiple knowledge-based systems. Besides, in recent years we have seen the emergence of the ontology language OWL [8] as a de-facto standard for knowledge and data representation on the web. For the purpose of domain knowledge representation, OWL (DL) is a more adequate language than (say) XML, since not only it promotes the separation of content and presentation, but it also provides support for the features (ii) and (iii) aforementioned. We reflect on the work reported here to highlight this, by contrasting it to our experience in using XML to represent electrocardiogram (ECG) data in a telemonitoring context-aware application [6].

The ECG [4] is the most frequently applied test for measuring heart activity in Cardiology. The analysis of the ECG waveform can help to identify a wide range of heart illnesses, which are distinguished by specific modifications on the ECG elementary forms. The ECG data can be presented through sound, text or chart image. The latter, however, is the most used media for exhibiting it, since heart beat patterns can be better recognized visually (by humans) in a graphical chart, see Figure 1. The ECG provides a mapping to the heart electrical activity taking place at the microscopic level. Understanding heart bioelectric phenomena is a demanding task from a cognitive point of view, due to its abstract nature and the requirements it poses in terms of spatial perception. That is why simulation-based visualization can make this less laborious. With this in mind, Wünsche states that [15]:

"Visualization is an attempt to simplify these tasks according to the motto: an image says more than a thousand words."

This paper then introduces the use of a lightweight ontology for reasoning and visualization of heart electrophysiology on the web. As elaborated in [16], this lightweight ontology is an implementation in OWL DL and its SWRL extension [9] of a reference ontology of heart electrophysiology (OHE) [5]. It then serves as a knowledge base for a web application. This application exploits user-interaction by processing clicks either on an ECG chart or on a heart-like image whereby heart phenomena are illustrated via animation. As a result of such clicks a reasoning engine draws logical inferences that control the animation. As we

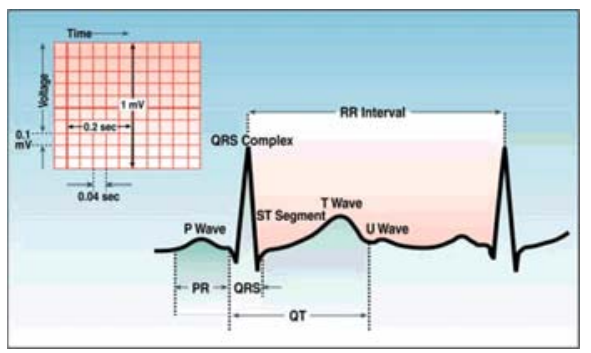

Figure 1. The normal ECG. 
discuss further on, this application should offer support for interactive learning in heart electrophysiology.

After discussing the use of web animations for education in section 2, we elaborate in section 3 on some benefits achieved in using OWL DL + SWRL for representing domain knowledge in web applications; section 4 , in turn, introduces the lightweight OHE; section 5 discusses how the ontology is used in the interactive web application proposed here, and section 6 report experiments to evaluate the application performance; finally, in section 7 some conclusions are provided.

\section{EDUCATIONAL WEB ANIMATIONS}

We have been inspired by García et al. in using flash animations for education [3]. In that article, the authors present several experiments that evidence benefits achieved in using animations to aid learning of Descriptive Geometry. Similarly, we believe that flash animations should offer support for exploratory learning in heart electrophysiology. By interacting with such web media, students could actively explore the ECG and the heart electrical system in a goal-oriented constructive process. They then could actually visualize the electrical currents generated by the heart pacemaker cells.

The two most common technologies to create animations in the web are Macromedia Flash ${ }^{1}$ and Scalable Vector Graphics $(\mathrm{SVG})^{2}$. The main difference between them lies in the temporal approach. While in Flash animations the computation is done frame-by-frame, SVG is time-based. This allows the animation to be free of frame rate, affording better performance for heavy animations. Flash, on the other hand, provides animation files (swf) of short size [3]. Although it requires plug-in to be processed by browsers, it is so widespread that according to estimates $97,4 \%$ of the users around the web can see SWF animations [3]. At a first glance, we have chosen flash to present both the ECG chart and the heart electrical system simulation. The simulation responds to events generated according to results computed by the reasoning engine.

\section{BENEFITS OF USING OWL DL + SWRL}

Although OWL DL + SWRL has serious limitations in terms of expressivity, it has noteworthy features for advancing applications on the web. First of all, being a W3C recommendation is a step forward in fostering interoperability between web applications. Secondly, this language has the feature of keeping domain knowledge and data aside from application code. The same ontology can then be reused by different applications. Indeed, reuse is also favored in OWL DL + SWRL with respect to the evolution of a given ontology. The reason is that the epistemic treatment of queries (asking about what is known) in OWL is designed to mirror the open-world assumption [12]. That is, what is not explicitly stated in the knowledge base is not considered false, leaving then the unknown open to further extensions. Finally, OWL DL + SWRL is designed to afford automated reasoning for deriving new information from existing information in the knowledge base. This feature is not possible by using, e.g., $\mathrm{XML}$, which is probably the most used language to data representation on the web nowadays. Such reasoning feature is

\footnotetext{
${ }^{1}$ Macromedia Flash website: <http://www.adobe.com/products/flash/>

${ }^{2}$ SVG website: <http://w3.org/Graphics/SVG>
}

also useful for checking logical consistency and reclassifying classes and individuals at run-time.

\section{THE LIGHTWEIGHT OHE ONTOLOGY}

The parts of OHE which are used in the web application further introduced in this paper are the sub-ontologies of (i) heart, (ii) bioelectric phenomena and (iii) ECG. As traditionally in Ontology Engineering, the scope and competence of OHE is defined by means of competence questions (CQ). By taking the end-application requirements into account, the following CQs (see [16]) are to be addressed.

CQ1. What conditions must be satisfied for the heart to play the role of a blood pump?

CQ7. What does the P wave represent in the ECG waveform?

CQ8. What does the QRS complex represent in the ECG waveform?

CQ1 is addressed by the first-order logic formulae A1, A2 and A3:

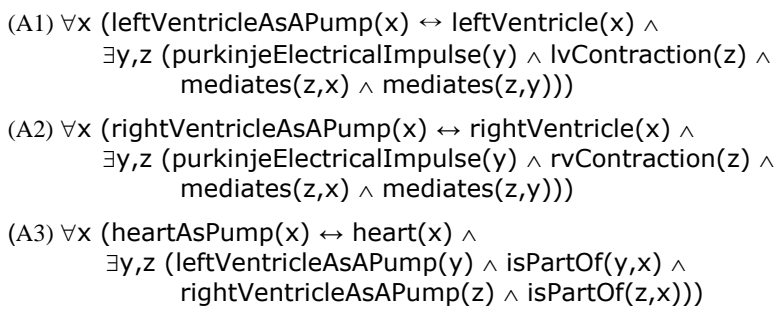

The formula A1 states that a left ventricle works as a pump if and only if there exists a purkinje electrical impulse triggering the contraction of this left ventricle. Similarly, A2 enunciates the necessary and sufficient conditions for a right ventricle to work as a pump. Finally, A3 states that a heart works as a pump if and only if it has both the left and right ventricles working as pumps. A3 is implemented in OWL DL as follows.

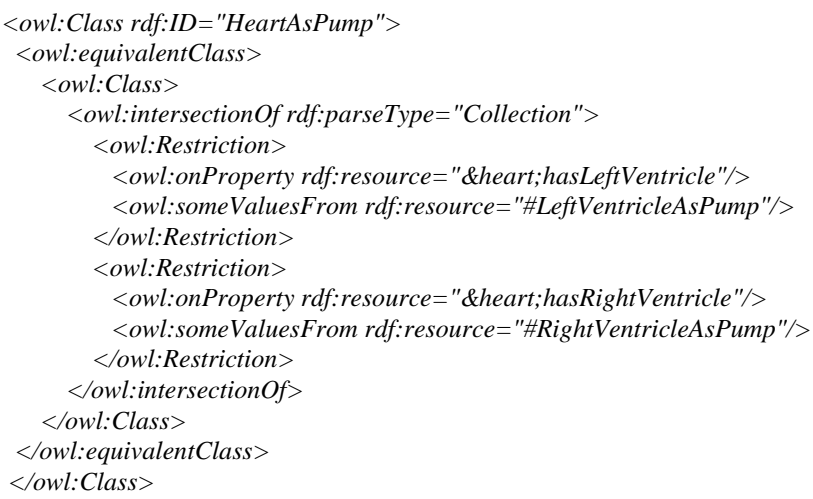

CQ7 and CQ8 in turn are concerned with the explicit representation of the meaning of the ECG elementary forms. That is, which sort of bioelectric phenomena are mapped by these ECG patterns (i.e., $\mathrm{P}$ wave and QRS complex). This is expressed by the formulae A4 and A5 below.

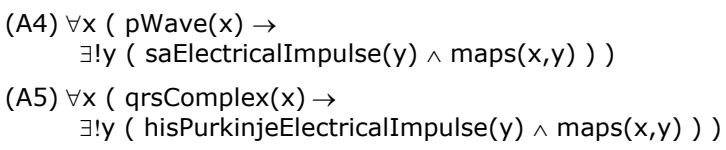

As it is enunciated by A4, the P wave maps the sinoatrial (SA) electrical impulse. The QRS complex in turn gives a trace of the 
His-Purkinje electrical impulse (cf. A5). In other words, the $\mathrm{P}$ wave and the QRS complex ECG elementary forms represent the bioelectric phenomena just mentioned in the ECG waveform. Moreover, it is worthwhile to say that the SA impulse triggers the atrial contraction and the His-Purkinje impulse (while passing throughout the Purkinje fibers) triggers the ventricular contraction. By considering the context of the end-application as well as the limitation of SWRL in expressivity, the formula A5 has been adapted to the following SWRL rule.

(R1) ecgTbox:NormalQRScomplex(?qrs) $\wedge$ ecgTbox:isSelected(?qrs, true) $\wedge$ bioElectric: PurkinjeElectricalImpulse(HisPurkinjeImpulse-0) $\rightarrow$ ecgTbox:maps(?qrs, HisElectricalImpulse-0)

When R1 is fired, it is inferred the relation maps correlating a QRS complex instance selected in the web application to a generic instance of His-Purkinje impulse. A more detailed description of how this rule and the rest of the ontology are used in the web application is given in what follows.

\section{THE WEB APPLICATION}

The web application proposed here has been implemented in Java by using the GWT framework ${ }^{3}$. GWT affords a client-server architecture in which asynchronous Remote Procedure Calls (RPC) take place. For handling the OWL ontology, we have used the Java APIs Jena [10] and Pellet [13]. Jena is used here mainly for providing to Pellet an access to the OWL ontology loaded in memory. Thus, the reasoning itself is fully supported by Pellet. The choice for the latter was due to its interesting characteristics reported in the literature. Pellet is efficient, customizable and can generate reasoning log information. Moreover, it also holds decidability even using SWRL rules (if they qualify as DL-safe [11]) as well as consistency validation between OWL restrictions and facts produced by SWRL rules.

The application allows three basic user interactions (see Figure 2):

(I1) selection (always by clicking) of an ECG record sample: this loads the record waveform into the ECG chart.

(I2) selection of a point on the ECG chart: this enables the reasoning engine to answer which ECG pattern is associated with this point. In case it is not located in any ECG elementary form (e.g. if it is in the ECG baseline, which corresponds to the heart resting state), just a message is shown in the log box; otherwise (i.e., the clicked point is located in an ECG elementary form), two events are triggered: (i) the chart is reloaded for emphasizing the identified pattern; and (ii) the bioelectric phenomenon is simulated in the heart-like image.

(I3) selection of a specific point of the heart-like image; this enables (i) the simulation of the bioelectric phenomenon correspondent to this point; and (ii) also reloads the ECG chart, but emphasizing the ECG pattern correlated to the point in all the cycles ${ }^{4}$ appearing in the ECG waveform.

The ontology is used by the application as follows. Concerning I1, the record sample mentioned is stored in an OWL file. This file can be viewed as an A-Box of the ontology ${ }^{5}$, since it contains

\footnotetext{
${ }^{3}$ Google Web Toolkit website: <http://code.google.com/webtoolkit/>

${ }^{4}$ These cycles constitute the heart beats.

5 An A-Box (or Assertional Box) in a DL-based ontology is the collection of the so-called facts (or instances of concepts and relations) in that ontology.
}

the facts (or individuals) that characterize one particular ECG record sample. Thus, instead of representing such an ECG record sample in a data format such as XML, or even binary format, we could take advantage of the expressivity of OWL for doing that. The added value is highlighted, e.g., in query-answering ECG records' related information, since the required knowledge is explicit in the ontology. I2, in turn, constitutes the main feature of the application. It is strongly based on the reasoning features. The user action of clicking a specific point of the ECG waveform implies the assertion of a new fact. This fact then enables the rule $\mathrm{R} 1$ to be fired if the point is associated with a normal ECG elementary form. In this case, once R1 is fired, the proper OWL restrictions are taken into account to indicate the inference results. Finally, I3 demands the ontology analogously to I1. It relies on the ontology A-Box to emphasize the proper elementary forms in all the cycles in which they occur.

All this can be illustrated by the situation shown in Figure 2. The user click was on the second cycle of the waveform. The server then has to fetch the ECG pattern associated with the clicked point by querying the ontology. As soon as the server has recognized it as a QRS complex in the Cycle 2, one fact indicating that the QRScomplex 2 was clicked is asserted into the ontology A-Box. The reasoning concerning this QRScomplex 2 is then performed. Since it is a normal QRS complex, R1 is fired. As a result, the heart behavior associated to the elementary form is inferred and described in the log box. The ECG chart is then reloaded showing only the QRS complex in the second cycle emphasized. Finally, the correlated heart electrical phenomenon is simulated through the flash animation (see Figure 2). All these correlations between ECG patterns and heart electrical phenomena are thus made explicit to the human observer.

\section{EXPERIMENTS}

In this section, we report experiments conducted in order to evaluate the performance of the proposed applicaton. They were performed using the application server Apache Tomcat 6.0.16 on a Microsoft Windows machine featuring AMD Turion $1.8 \mathrm{GHz}$ and $1 \mathrm{~GB}$ of main memory. The web application is implemented in Java 1.6.0_02 by using GWT 1.4, Jena-2.5.5 and Pellet-1.5.2.

The timing measurements for $\mathbf{I} \mathbf{2}$ are the foremost in relevance. The reason is that they give an outlook of the application performance bottleneck: the OWL DL + SWRL-based automated reasoning. The first results are encouraging. As shown in Table 1, the reasoning takes just $1.6 \mathrm{~ms}$, on average; while information retrieval is also not so time-consuming (2689 ms, on average).

Table 1. Timing measurements (in $\mathrm{ms}$ ) for $\mathrm{I} 2$

\begin{tabular}{|c|c|c|c|}
\hline Event & Mean & Median & Stand. Deviation \\
\hline Click processing & 0 & 0 & 0 \\
\hline RPC call & 7.9 & 8.3 & 7.5 \\
\hline Loading OWL files & 200 & 58.8 & 179.5 \\
\hline Fetching ECG pattern & 0 & 0 & 0 \\
\hline Reasoning & 1.6 & 5.1 & 0 \\
\hline Information retrieval & 2689 & 2860.4 & 2422 \\
\hline RPC callback & 14 & 4.9 & 15.5 \\
\hline Callback processing & 46.9 & 32.1 & 39.5 \\
\hline Total time & 2959.4 & 2844.3 & 2742 \\
\hline
\end{tabular}

For the case of 76 classes, 85 properties and 222 individuals, the information retrieval time stays up to $5111 \mathrm{~ms}$. Nonetheless, there are multiple ways of retrieving the same piece of information in 


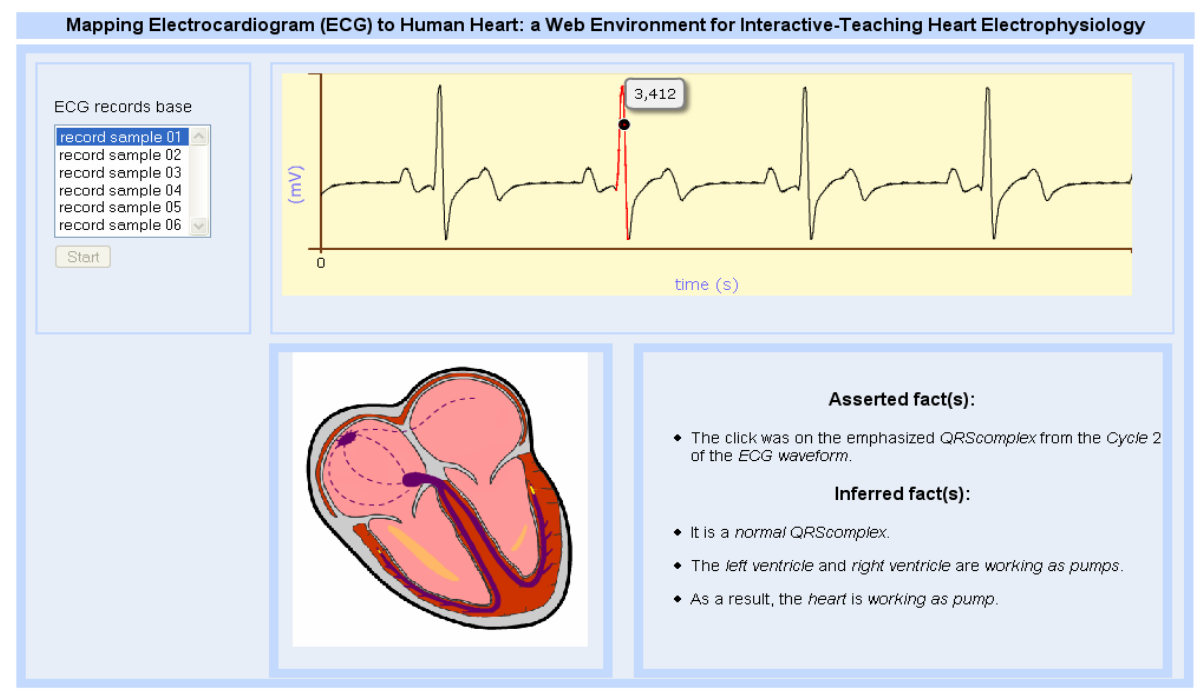

Figure 2. The ontology-based web application.

Pellet/Jena, and we have not yet given attention to optimization. The DL expressivity of the lightweight OHE is ALCHIN(D) (see [1] for expressivity details of this DL). Depending on the expressivity, different completion strategies are used by Pellet. RuleStrategy has been used in this case (it is the default one in the presence of rules). With respect to the media, the ECG chart and heart animation SWF files have $64 \mathrm{~kb}$ and $35 \mathrm{~kb}$ respectively. These sizes are quite small for the server-to-client transmission.

\section{CONCLUSIONS}

We introduce in this paper the use of a lightweight ontology of heart electrophysiology in a web application. This application allows the visualization of ECG records and heart bioelectric phenomena, and it can be used to offer support for learning on the web. As discussed before in this text, the literature has evidenced the assumption that ontology is a suitable formalism to represent biomedical sub-domains. This paper, however, is an attempt to provide evidence for the following. More than serving as a reusable (data) representation, an OWL DL + SWRL ontology can be used as a knowledge base for automated reasoning in these (henceforth) semantically-enhanced biomedical applications. Although this principle is potentially valid in other domains, its benefits become particularly evident in handling complex domains such as Biomedicine. The reason is that such domains usually call for a stronger effort in knowledge representation. The output, however, can then be reused across multiple visualization media and/or applications.

\section{ACKNOWLEDGEMENTS}

This research is partially supported by the projects MODELA and INFRA-MODELA funded by the Brazilian funding agencies FACITEC and FAPES, respectively.

\section{REFERENCES}

[1] Baader, F., et al. (eds) 2003. The Description Logic Handbook: Theory, Implementation, and Applications. Cambridge Univ. Press.

[2] Burgun, A. 2006. A Desiderata for Domain Reference Ontologies in Biomedicine. Journal of Biomedical Informatics, 39(3): 307-313. [DOI: 10.1016/j.jbi.2005.09.002]

[3] García, R. et al. 2007. Interactive Multimedia Animation with Macromedia Flash in Descriptive Geometry Teaching. Computers \&
Education, Elsevier, 49(3):615-639. [DOI: 10.1016/j.compedu.2005.11.005]

[4] Geselowitz, D. 1989. On the Theory of the Electrocardiogram. Proceedings of the IEEE, 77(6): 857-876. [DOI: 10.1109/5.29327]

[5] Gonçalves, B., et al. 2007. An Electrocardiogram (ECG) Domain Ontology. In Proc. of the $2^{\text {nd }}$ Workshop on Ontologies and Metamodels for Software and Data Engineering, João Pessoa, Brazil.

[6] Gonçalves, B., Pereira Filho, J., Andreão, R. 2008. ECGAWARE: an ECG Markup Language for Ambulatory Telemonitoring and Decision Making Support. In Proc. of the International Conf. on Health Informatics (HEALTHINF’08), Funchal, Portugal.

[7] Hahn, U., Schulz, S. (eds). 2007. Ontological Foundations for Biomedical Sciences. Artificial Intelligence in Medicine, 39(3): 179182. [DOI: 10.1016/j.artmed.2006.12.006]

[8] Horrocks, I. et al. From SHIQ and RDF to OWL: The Making of a Web Ontology Language. Journal of Web Semantics, 1(1): 7-26, 2003. [DOI: 10.1016/j.websem.2003.07.001]

[9] Horrocks, I. et al. 2005. OWL Rules: a Proposal and Prototype Implementation. Journal of Web Semantics, 3(1): 23-40. [DOI: 10.1016/j.websem.2005.05.003]

[10] McBride, B. 2002. Jena: a Semantic Web Toolkit. IEEE Internet Computing, 6(6): 55-59. [DOI: 10.1109/MIC.2002.1067737]

[11] Motik, B. et al. 2005. Query Answering for OWL-DL with Rules. Journal of Web Semantics, 3(1):41-60. [DOI:10.1016/j.websem.2005.05.001]

[12] Patel-Schneider, P.,Horrocks, I. 2007. A Comparison of Two Modelling Paradigms in the Semantic Web. Journal of Web Semantics, 5(4):240-250. [DOI: 10.1016/j.websem.2007.09.004]

[13] Sirin, E. et al. 2007. Pellet: a Practical OWL-DL Reasoner. Journal of Web Semantics, 5(2): 51-53. [DOI: 10.1016/j.websem.2007.03.004]

[14] Smith, B. et al. 2007. The OBO Foundry: Coordinated Evolution of Ontologies to Support Biomedical Data Integration. Nature Biotechnology, 25(11): 1251-1255. [DOI: 10.1038/nbt1346]

[15] Wunsche, C. 2003. A Toolkit for Visualizing Biomedical Data Sets. In Proc. of the 1st International Conf. on Computer Graphics and Interactive Techniques in Australasia and South East Asia, Melbourne, Australia. [DOI: 10.1145/604471.604505]

[16] Zamborlini, V., Gonçalves, B., Guizzardi, G. 2008. Codification and Application of a Well-Founded Heart-ECG Ontology. In Proc. of the $3^{\text {rd }}$ Workshop on Ontologies and Metamodels for Software and Data Engineering (WOMSDE’08), Campinas, Brazil. 